\title{
DISCOVERY OF THE MASSIVE OVERCONTACT BINARY VFTS 352: EVIDENCE FOR ENHANCED INTERNAL MIXING
}

\author{
L. A. Almeida ${ }^{1,2}$, H. SAna ${ }^{3,4}$, S. E. de Mink ${ }^{5}$, F. Tramper ${ }^{5}$, I. Soszyński ${ }^{6}$, N. Langer ${ }^{7}$, R. H. Barbá ${ }^{8}$, M. Cantiello 9 , \\ A. Damineli ${ }^{2}$, A. De Koter ${ }^{5,10}$, M. Garcia ${ }^{11}$, G. Gräfener ${ }^{12}$, A. Herrero ${ }^{13,14}$, I. Howarth ${ }^{15}$, J. Maíz Apellániz ${ }^{11}$, \\ C. Norman ${ }^{1}$, O. H. RamíreZ-Agudelo ${ }^{5}$, AND J. S. VinK ${ }^{12}$ \\ ${ }^{1}$ Department of Physics \& Astronomy, Johns Hopkins University, Bloomberg Center for Physics and Astronomy, Room 520, 3400N Charles St, USA; \\ leonadodealmeida.andrade@gmail.com \\ ${ }^{2}$ Instituto de Astronomia, Geofísica e Ciências, Rua do Matão 1226, Cidade Universitária São Paulo, SP, 05508-090, Brasil \\ ${ }^{3}$ European Space Agency/Space Telescope Science Institute, 3700 San Martin Drive, Baltimore, MD 21218, USA \\ ${ }^{4}$ Institute of Astronomy, Ku Leuven, Celestijnlaan 200D, 3001 Leuven, Belgium \\ 5 Anton Pannenkoek Astronomical Institute, University of Amsterdam, 1090 GE Amsterdam, The Netherlands \\ ${ }^{6}$ Warsaw University Observatory, Al. Ujazdowskie 4, 00-478 Warszawa, Poland \\ ${ }^{7}$ Argelander-Institut fr Astronomie, der Universität Bonn, Auf dem Hgel 71, D-53121 Bonn, Germany \\ ${ }^{8}$ Departamento de Física y Astronomía, Universidad de La Serena, Av. Cisternas 1200 Norte, La Serena, Chile \\ ${ }_{9}$ Kavli Institute for Theoretical Physics, University of California, Santa Barbara, CA 93106, USA \\ ${ }^{10}$ Instituut voor Sterrenkunde, Universiteit Leuven, Celestijnenlaan 200 D, 3001, Leuven, Belgium \\ ${ }^{11}$ Departamento de Astrofísica, Centro de Astrobiología (INTA-CSIC), campus ESA, apartado postal 78, 28 691 Villanueva de la Cañada, Madrid, Spain \\ ${ }^{12}$ Armagh Observatory, College Hill, Armagh, BT61 9DG, Northern Ireland, UK \\ ${ }^{13}$ Instituto de Astrofísica de Canarias, C/ Vía Láctea s/n, E-38200 La Laguna, Tenerife, Spain \\ ${ }^{14}$ Departamento de Astrofísica, Universidad de La Laguna, Avda. Astrofísico Francisco Sánchez s/n, E-38071 La Laguna, Tenerife, Spain \\ 15 Department Physics Astronomy, University College London, Gower Street, London WC1E 6BT, UK \\ Received 2014 December 12; accepted 2015 September 2; published 2015 October 13
}

\begin{abstract}
The contact phase expected to precede the coalescence of two massive stars is poorly characterized due to the paucity of observational constraints. Here we report on the discovery of VFTS 352, an O-type binary in the 30 Doradus region, as the most massive and earliest spectral type overcontact system known to date. We derived the 3D geometry of the system, its orbital period $P_{\mathrm{orb}}=1.1241452(4)$ day, components' effective temperatures$T_{1}=42540 \pm 280 \mathrm{~K}$ and $T_{2}=41120 \pm 290 \mathrm{~K}$ - and dynamical masses- $M_{1}=28.63 \pm 0.30 M_{\odot}$ and $M_{2}=28.85 \pm 0.30 M_{\odot}$. Compared to single-star evolutionary models, the VFTS 352 components are too hot for their dynamical masses by about 2700 and $1100 \mathrm{~K}$, respectively. These results can be explained naturally as a result of enhanced mixing, theoretically predicted to occur in very short-period tidally locked systems. The VFTS 352 components are two of the best candidates identified so far to undergo this so-called chemically homogeneous evolution. The future of VFTS 352 is uncertain. If the two stars merge, a very rapidly rotating star will be produced. Instead, if the stars continue to evolve homogeneously and keep shrinking within their Roche Lobes, coalescence can be avoided. In this case, tides may counteract the spin down by winds such that the VFTS 352 components may, at the end of their life, fulfill the requirements for long gamma-ray burst (GRB) progenitors in the collapsar scenario. Independently of whether the VFTS 352 components become GRB progenitors, this scenario makes VFTS 352 interesting as a progenitor of a black hole binary, hence as a potential gravitational wave source through black hole-black hole merger.
\end{abstract}

Key words: binaries: close - binaries: eclipsing - binaries: spectroscopic - stars: early-type stars: individual (VFTS 352) - stars: massive

\section{INTRODUCTION}

Massive stars are one of the most important cosmic engines driving the evolution of galaxies throughout the history of the universe. In the Milky Way, most massive stars are born as part of a binary system with an orbital period $P_{\text {orb }}<4$ year (Mason et al. 2009; Sana et al. 2012), i.e., close enough to interact during their lifetime through mass-exchange or coalescence (Podsiadlowski et al. 1992). Such binary interactions have important consequences for the subsequent evolution of the components of the system and lead to key astrophysical phenomena such as double compact binaries, hydrogen-deficient core-collapse supernovae and gamma-ray bursts (GRBs). The high frequency of close binaries further challenges the accepted predominance of the single-star evolutionary channel. Binary interaction may happen early in the evolution of the stars. According to the massive star multiplicity properties derived from galactic young open clusters (Sana et al. 2012), 40\% of all O-type stars may interact with a nearby companion before leaving the main sequence and over half these main sequence interactions (about a quarter of all stars born as O type) will lead to a deep contact phase of which the most likely outcome will be coalescence. Such a process modifies the mass function of massive star clusters, and may help to create some of the most massive stars known (Crowther et al. 2010; Schneider et al. 2014a).

Despite possibly affecting almost one quarter of all O-type stars, the deep contact phase is one of the least understood phases of massive binary evolution. This results from the complex interplay between a variety of physical processes, including mass exchange, tidal locking, intense mutual illumination, internal mixing, angular momentum loss, as well as possible adjustments to the stars internal structure (Pols 1994; Wellstein et al. 2001; de Mink et al. 2007).

Our limited understanding also stems from the almost complete absence of observational constraints. Observationally, 


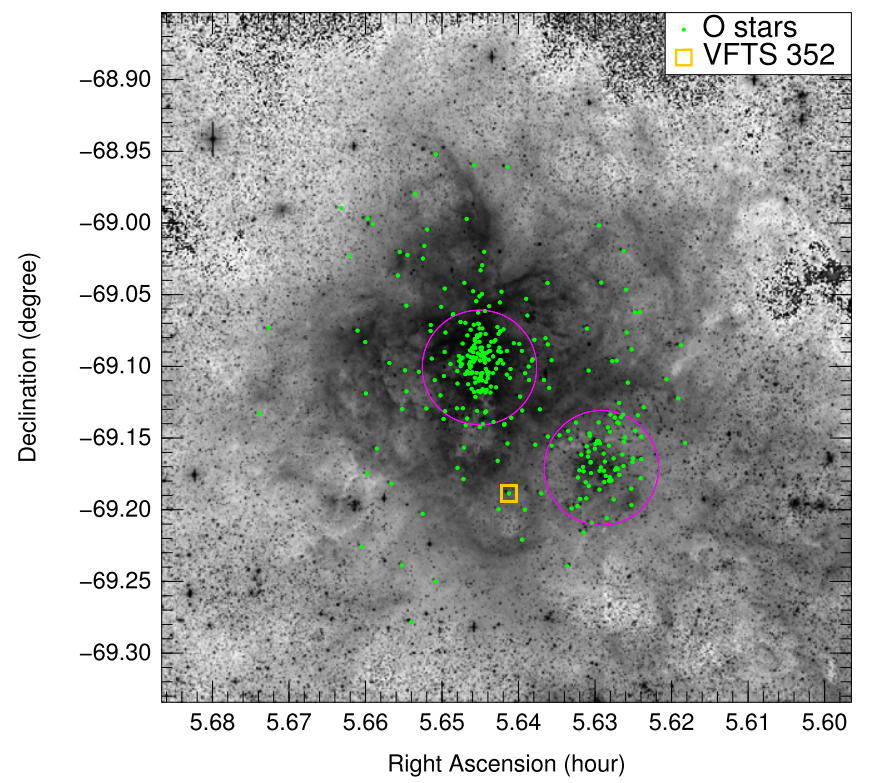

Figure 1. Location of VFTS 352 in the 30 Dor field of view. All O-type stars observed by the VFTS are also marked. The two circles indicate the locations of NGC 2070 (at the center) and NGC 2060 (to the south-west).

these interacting binaries are expected to reveal themselves as overcontact binaries, i.e., an orbital configuration in which both components are overfilling their Roche lobes (Wilson 2001). However, only three O-type overcontact binaries are known to date (see Section 4.1).

Located in the Tarantula Nebula (30 Dor, Figure 1) in the Large Magellanic Cloud (LMC), VFTS 352 is a double-lined spectroscopic binary identified by the VLT-FLAMES Tarantula Survey (VFTS, Evans et al. 2011; Sana et al. 2013). Classified as $\mathrm{O} 4.5 \mathrm{~V}(\mathrm{n})((\mathrm{fc})) \mathrm{z}+\mathrm{O} 5.5 \mathrm{~V}(\mathrm{n})((\mathrm{fc})) \mathrm{z}$ (Walborn et al. 2014), both companions present rotationally broadened $\mathrm{H}$ I, He I and He II absorption lines. In this study we present the results of the analysis of an 18-month spectroscopic monitoring of VFTS 352 combined with $\sim 12$ years of Optical Gravitational Lensing Experiment (OGLE)-III and IV photometry (Udalski et al. 2008).

\section{OBSERVATIONS AND DATA REDUCTION}

\subsection{Spectroscopy}

New spectroscopic data of VFTS 352 were collected as part of the Tarantula Massive Binary Monitoring program (PI: Sana) at the ESO Very Large Telescope (VLT). 32 spectroscopic epochs were obtained from 2012 October to 2014 March using the FLAMES-GIRAFFE multi-object spectrograph and the LR02 grism, providing continuous coverage of the 3950-4550 A region with a spectral resolving power $\lambda / \Delta \lambda$ of 6,400 . Three sets of back-to-back exposures were taken to ensure optimal cosmic-ray removal. The six LR02 spectra obtained as part of the VFTS were further included in our analysis.

The data reduction was performed using the ESO CPL GIRAFFE pipeline v.2.12.1. It involved the standard steps of bias subtraction, flat-field structure removal, extraction, and wavelength calibration. Additional steps of sky correction and normalization were applied following the procedures described in Evans et al. (2011) and Sana et al. (2013), respectively. Figure 2 shows examples of normalized individual spectra.

\subsection{Photometry}

$V$ - and I-band time-resolved photometry were obtained by the OGLE-III and IV projects (Udalski et al. 2008). The temporal coverage of the OGLE-III and IV data is of $\sim 7.5$ and 5.2 years, respectively, with an accuracy of a few mmag. There is a small shift ( $\sim 0.01 \mathrm{mag})$ in brightness between OGLE-III and OGLE-IV light curves due to independent calibration which has an accurancy of about $0.02 \mathrm{mag}$ (Udalski et al. 2008). The total number of VFTS 352 measurements available in the $V$ - and $I$-bands is 90 and 760 . Both light curves show ellipsoidal variations with semi-amplitude of $\sim 0.1 \mathrm{mag}$ indicating that at least one component of the system is filling its Roche lobe or is close to doing so.

\section{ANALYSIS AND RESULTS}

\subsection{Radial Velocity Measurements}

The radial velocities of the VFTS 352 components were obtained following the procedure described in Sana et al. (2013). In brief, we simultaneously adjusted the $\mathrm{He}_{\mathrm{I}} \lambda \lambda 4388$, 4471 and He II $\lambda \lambda 4200,4541$ lines at all epochs using Gaussian profiles. The shape of the Gaussian profile for each spectral line and each component is kept constant at all epochs, allowing for enhanced robustness of the fitting at phases where the lines are heavily blended or where the $\mathrm{S} / \mathrm{N}$ is poorer. The obtained RVs and $1 \sigma$ error bars are available at the Centre de Données astronomiques de Strasbourg ${ }^{15}$ together with the journal of the observations. The best-fit RV-only orbital solution yields semiamplitudes of $K_{1}=324.9 \pm 5.8 \mathrm{~km} \mathrm{~s}^{-1}$ and $K_{2}=315.6 \pm$ $6.2 \mathrm{~km} \mathrm{~s}^{-1}$ for the primary and secondary components. In the next section, the RV-only solution that we obtain is used as a starting point for the combined photometric and spectroscopic orbital solution.

\subsection{Simultaneous Modeling of Light Curves and Radial Velocities}

To obtain the geometrical and physical parameters of VFTS 352, we simultaneously adjusted synthetic light and radial velocity curves generated by the Wilson Devinney code (WDC, Wilson \& Devinney 1971) to the $V$ - and I-band OGLEIV light curves and the VLT/FLAMES radial velocity curves. We follow the procedure presented in Almeida et al. (2012) to search for the global solution. In essence, the WDC is used as a function to be optimized by the genetic algorithm PIKAIA (Charbonneau 1995) followed by a Markov chain Monte Carlo (MCMC) procedure (Gilks et al. 1996) to sample the parameters and obtain their uncertainties.

To restrict the size of the explored parameter space, we use priors obtained from our initial radial velocity solution (Section 3.1) and from the known spectral type of the components (Walborn et al. 2014). We constrain the mass ratio $\left(q=M_{2} / M_{1}\right)$ between $0.95<q<1.05$, and effective temperatures, $40,900<T_{\text {eff, } 1}<44,900$ and $38,900 \mathrm{~K}<$ $T_{\text {eff,2 }}<42,900 \mathrm{~K}$, with 42,900 and 40,900 K (Walborn et al. 2014) as a starting point for the primary and secondary respectively. We also keep fixed the distance $d=50 \mathrm{kpc}$ and the $V$-band extinction $A_{v}=1.109 \pm 0046$ (Maíz Apellániz et al. 2014). As the light curves show ellipsoidal variation and $q \sim 1$, we try to model the data with overcontact, contact, and

\footnotetext{
15 http://vizier.u-strasbg.fr/
} 


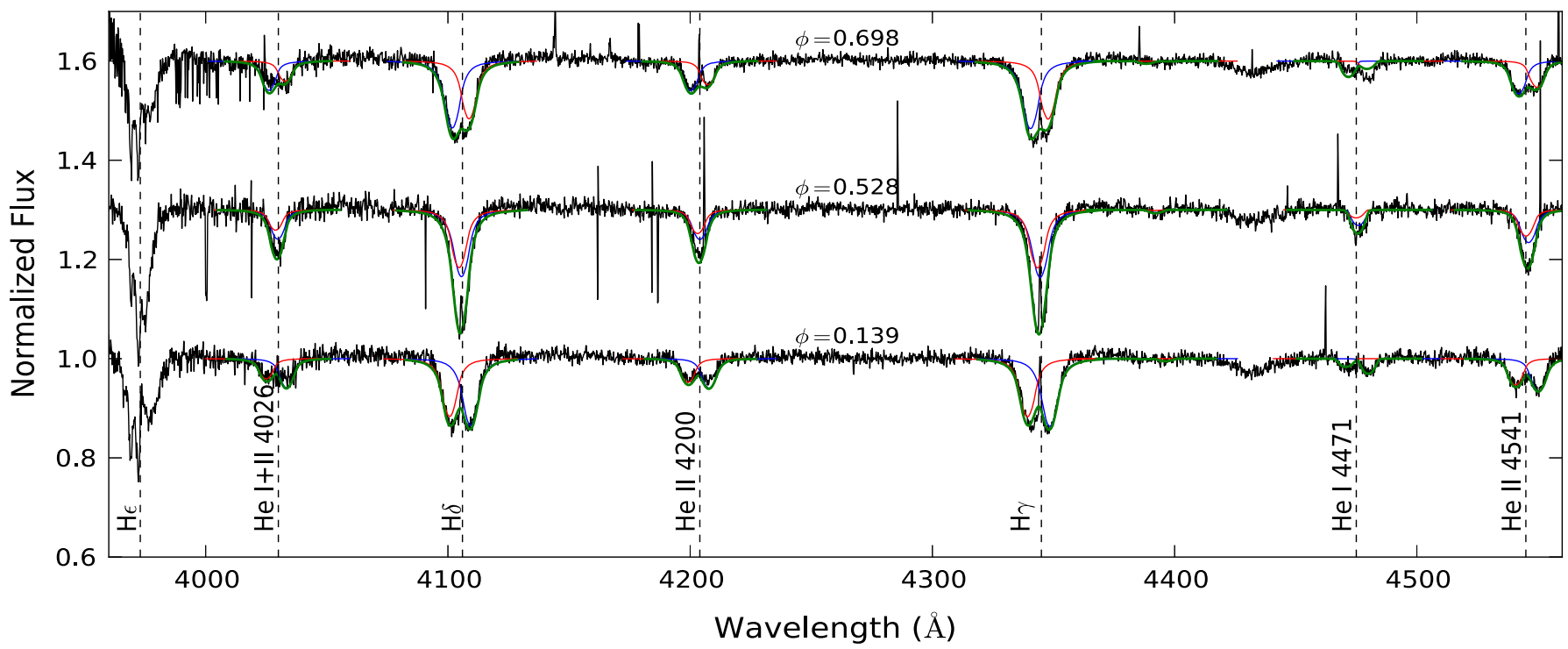

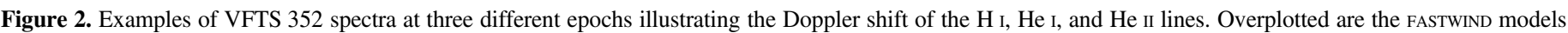

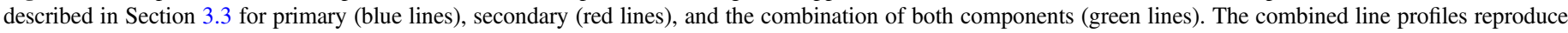
the observed binary spectrum very well.

semi-detached configurations, but only the overcontact model provides a plausible solution (see the top panel of Figure 3). In all these models, non-Keplerian effects (tidal distortion of the stars) are taken into account.

To account for the temperature difference between the components (see Section 3.3), we use the mode 3 of the WDC which has no constrain in the temperature, bolometric albedo, gravity brightening, and limb darkening coefficients of the secondary star. WDC converts the mean surface effective temperatures, $T_{1}$ and $T_{2}$, in polar temperature using Equation (8) of Wilson (1979) and then computes the local temperature using gravity brightening law and polar temperature. The linear limb darkening coefficients were computed for both stars as a function of $T_{\text {eff }}$ and $\log g$. In this purpose, the coefficients calculated by Díaz-Cordovés et al. (1995) and Claret et al. (1995) are automatically updated while the program is running. The best solution for VFTS 352 is shown in Figure 3 and the adjusted and derived parameters are listed in Table 1.

\subsection{Atmosphere Analysis}

We employ the line-blanketed non-local thermodynamic equilibrium atmosphere code FASTWIND (Puls et al. 2005) to model both components of VFTS 352. For our initial models we use the values for the radius, gravity and temperatures listed in Table 1 as input. We adopt the Vink et al. (2001) mass-loss rates based on the stellar parameters and an LMC metallicity and we use the scaling with the escape velocity $\left(v_{\mathrm{esc}}\right)$ to estimate the wind terminal velocity $\left(v_{\infty}=2.65 \times v_{\mathrm{esc}} ;\right.$ Kudritzki \& Puls 2000). Finally, we adopt rotation rates corresponding to a fully synchronized system, i.e., $v_{\text {rot }}=325 \mathrm{~km} \mathrm{~s}^{-1}$, and we use the orbital inclination value $(i)$ from Table 1 to compute the projected rotation velocity at the equator.

The model that we obtained (Figure 2) further takes into account the phase dependance of the brightness ratio of the two components and provides a satisfactory representation of the data. In addition to the physical parameters, we also investigate the helium surface abundance in the VFTS 352 spectra. While we could not find any clear signature of enrichment, we estimate that a surface enrichment corresponding to a mass fraction $X_{\mathrm{He}}$ less than $40 \%$ would be hard to detect with our data.

Figure 2 also reveals small variations in the intensity of the He I lines. Such a behavior is reminiscent of the Struve-Sahade effect (Bagnuolo et al. 1999; Linder et al. 2007) and possibly results from temperature structure on the (deformed) stellar surface (Palate \& Rauw 2012). Future spectral disentangling, taking into account the phase dependance flux-ratio of the object-may help to lower the present upper limit on the He surface abundance. Combined with phase-resolved spectral modeling, it would further help to ellucidate the nature of the He I lines line profile variability. These two aspects however lay beyond the scope of the present work.

\subsection{Orbital Period Variation}

To search for indication of variations in the orbital configuration of VFTS 352, we scrutinize its orbital period. We split the OGLE-III and IV I-band photometric data-which span 12.5 years-into 15 parts that we analyze separately using the WDC. As all the VFTS 352 parameters are well determined (see Table 1), we allow them to vary only within $\pm 1 \sigma$ from their best-fit value and we leave the orbital period as the only entirely free parameter. The MCMC procedure was used to sample the posterior distribution of the orbital period and obtain the best value as well as its uncertainty.

With a peak-to-peak variation of $\sim 2 \mathrm{~s}$ over a 12.5 year time span, the variation time scale of the VFTS 352 orbital period is approximately $P / \dot{P} \approx 0.6 \mathrm{Myr}$, see Figure 4 . Such a relatively long time scale suggests that the system may currenty be stable, thus the overcontact configuration relatively long lived. In that case, the relevant evolutionary time scale for the system is the nuclear time scale. The apparent stability of the VFTS 352 configuration would then favor an evolutionary stage that corresponds to the rather long-lasting overcontact phase predicted by Case A mass transfer scenarios (Pols 1994; Wellstein et al. 2001). 


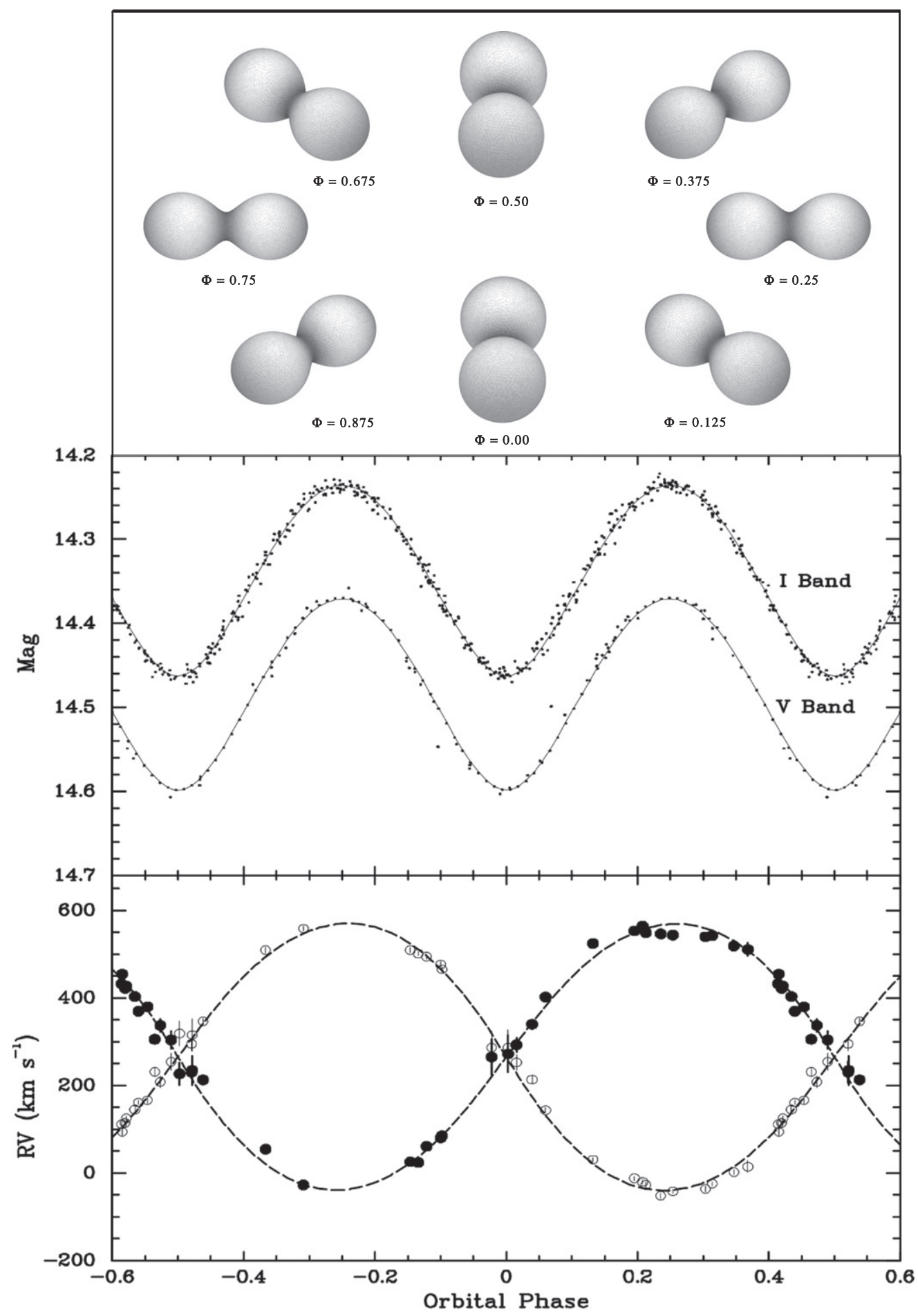

Figure 3. From the top to the bottom panels are: snapshots of VFTS 352 at few orbital phases, as viewed from an orbital inclination angle $i$ of $55^{\circ} .6$; and best WDC model simultaneously fitted to the $V$ - and $I$-band light curves and radial velocity curves, respectively.

\section{DISCUSSION AND CONCLUSION}

\subsection{Physical Properties}

The simultaneous analysis of the RV and light curves of VFTS 352 indicates that this system is in an overcontact configuration. While a number of massive contact binaries are known, i.e., systems where both stars just fill their Roche lobes (e.g., MY Cam and UW CMa, Leung \& Schneider 1978; Antokhina et al. 2011; Lorenzo et al. 2014) only a few overcontact O-type systems have been identified so far: TU Mus $\left(\mathrm{O} 8 V(n) z+\mathrm{B} 0 V(n) ; P_{\text {orb }}=1.38\right.$ day, Penny et al. 2008; Sota et al. 2014) and V382 Cyg (O7V + O7V; $P_{\text {orb }}=1.89$ day, Popper 1978; Değirmenci et al. 1999) in the Milky Way, and OGLE SMC-SC10 108086 (O7 V + O8 V; $P_{\text {orb }}=0.88$ day, Hilditch et al. 2005) in the Small Magellanic Cloud.

By comparing the stars' volumes with those of their Roche lobes, one can estimate the degree of (over)contact. For this 
Table 1

Best-fit Parameters Resulting from the Simultaneous Adjustment in the $I$ - and $V$-band Light Curves and Radial Velocity Curves of the Components

\begin{tabular}{lc}
\hline \hline Parameter & Value \\
\hline \multicolumn{2}{c}{ Adjusted Parameters } \\
\hline$P_{\text {orb }}($ day $)$ & $1.1241452 \pm 0.0000004$ \\
$T_{0}(\mathrm{HJD})$ & $2455261.119 \pm 0.003$ \\
$q=M_{2} / M_{1}$ & $1.010 \pm 0.010$ \\
$\Omega_{1}{ }^{\mathrm{a}}$ & $3.584 \pm 0.014$ \\
$\Omega_{2}{ }^{\mathrm{a}}$ & $3.584 \pm 0.014$ \\
$T_{1}(\mathrm{~K})$ & $42540 \pm 280$ \\
$T_{2}(\mathrm{~K})$ & $41120 \pm 290$ \\
$i\left(^{\circ}\right)$ & $55.60 \pm 0.20$ \\
$a^{\mathrm{b}}\left(R_{\odot}\right)$ & $17.55 \pm 0.06$ \\
$\gamma\left(\mathrm{km} \mathrm{s}^{-1}\right)$ & $262.8 \pm 1.2$ \\
\hline
\end{tabular}

\begin{tabular}{lc}
\hline \multicolumn{3}{c}{ Roche Radii } \\
\hline$R_{1}^{\text {pole }} / R_{\mathrm{L}}$ & $1.005 \pm 0.001$ \\
$R_{1}^{\text {side }} / R_{\mathrm{L}}$ & $1.070 \pm 0.001$ \\
$R_{1}^{\text {back }} / R_{\mathrm{L}}$ & $1.192 \pm 0.001$ \\
$R_{1}^{\text {mean }} / R_{\mathrm{L}}$ & $1.089 \pm 0.001$ \\
$R_{2}^{\text {pole }} / R_{\mathrm{L}}$ & $1.004 \pm 0.001$ \\
$R_{2}^{\text {side }} / R_{\mathrm{L}}$ & $1.067 \pm 0.001$ \\
$R_{2}^{\text {back }} / R_{\mathrm{L}}$ & $1.189 \pm 0.001$ \\
$R_{2}^{\text {mean }} / R_{\mathrm{L}}$ & $1.087 \pm 0.001$ \\
\hline
\end{tabular}

\begin{tabular}{lr}
\hline \multicolumn{3}{c}{ Derived Parameters } \\
\hline$M_{1}\left(M_{\odot}\right)$ & $28.63 \pm 0.30$ \\
$M_{2}\left(M_{\odot}\right)$ & $28.85 \pm 0.30$ \\
$R_{1}^{\text {mean }}\left(R_{\odot}\right)$ & $7.22 \pm 0.02$ \\
$R_{2}^{\text {mean }}\left(R_{\odot}\right)$ & $7.25 \pm 0.02$ \\
$\log g_{1}\left(\mathrm{~cm} \mathrm{~s}^{-2}\right)$ & $4.18 \pm 0.01$ \\
$\log g_{2}\left(\mathrm{~cm} \mathrm{~s}^{-2}\right)$ & $4.18 \pm 0.01$ \\
$M_{1}^{\text {bol }}$ & $-8.26 \pm 0.01$ \\
$M_{2}^{\text {bol }}$ & $-8.08 \pm 0.01$ \\
\hline
\end{tabular}

Note.

${ }^{\text {a }}$ Roche surface potential $-\Omega_{2}=\Omega_{1}$ in mode 3 of the WDC;

${ }^{\mathrm{b}}$ Binary separation.

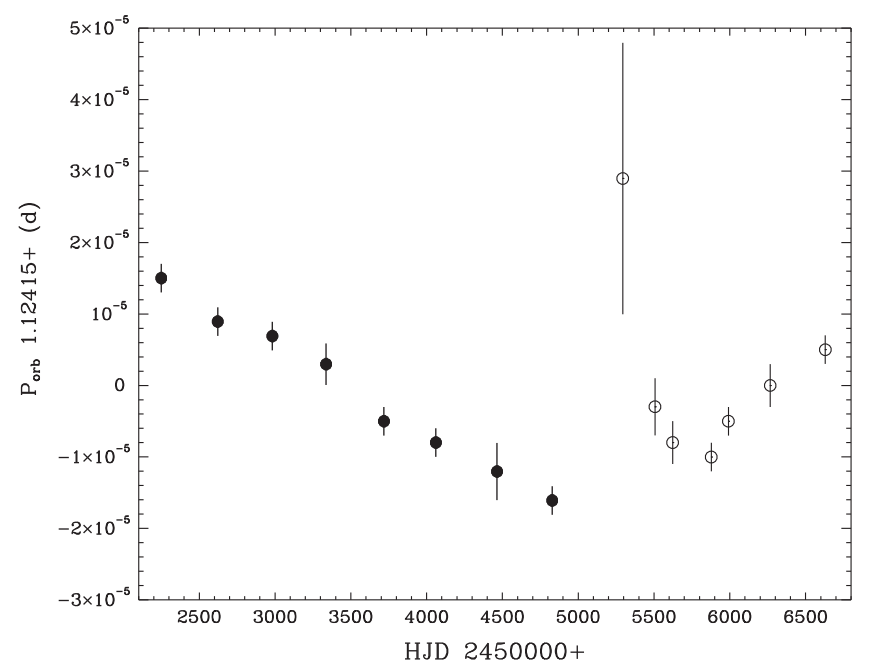

Figure 4. Orbital period variation of VFTS 352. The orbital period measurements obtained from OGLE-III and IV I-band photometric data are shown as filled and open circles respectively. purpose, we define the Roche volume (over)filling factor $f_{\mathrm{L}}$ as the ratio between the volume of the star and that of its Roche lobe. $f_{\mathrm{L}}$ can be approximated as

$$
f_{\mathrm{L}}=\left(\frac{R^{\text {mean }}}{R_{\mathrm{L}}}\right)^{3},
$$

with $R^{\text {mean }}$, the average stellar radius and $R_{\mathrm{L}}$, the effective Roche lobe radius that we compute using Eggleton's (1983) approximation. According to this definition, both components of VFTS 352 have $f_{\mathrm{L}} \approx 1.29$. Stars in TU Mus have $f_{\mathrm{L}} \approx 1.30$ and 1.24 while the components of V382 Cyg and OGLE SMC-SC10 108086 have $f_{\mathrm{L}} \approx 1.1$ and 1.7, respectively.

According to their spectral classifications, the VFTS 352 primary and secondary components have effective temperatures of about 42,900 and 40,900 K (Walborn et al. 2014). These values agree very well with the results of the light curves fitting $-T_{1}=42,540 \pm 280 \mathrm{~K}$ and $T_{2}=41,120 \pm 290 \mathrm{~K}$ - and are further consistent with the observed spectra of VFTS 352 (Section 3.3). The good agreement between the three methods suggests robust effective temperature estimates.

Figure 5 compares the location of the VFTS 352 components in the Hertzsprung-Russell diagram (HRD) with those of the other known overcontact binaries. It reveals that VFTS 352 is the earliest-type, hottest, and most massive overcontact binary known to date. VFTS 352 is also the second shortest orbital period system and the only one with a mass-ratio close to unity.

At 1 Myr, single stars of 28.63 and $28.85 M_{\odot}$ with initial spin rates $\approx 330 \mathrm{~km} \mathrm{~s}^{-1}$ are expected to have effective temperatures of 39,880 and 39,980 K (Brott et al. 2011). The VFTS 352 components are thus hotter by about 2700 and $1100 \mathrm{~K}$, respectively. As a confirmation, Figure 6 shows that the quasi-absence of $\mathrm{He}_{\mathrm{I}} \lambda \lambda 4143,4387$ and the weak He I $\lambda 4471$ in the primary spectrum cannot be reconciled with the temperature of $39,880 \mathrm{~K}$ that is expected given its mass, its spin rate and the Brott et al. (2011) models. To be noted, the FASTWIND models in Figures 2 and 6 are not fitted, but serve as a check that the light curve fitting result and, in particular, its absolute temperature scale, are fully consistent with the object spectrum. Interestingly, the primary is the most luminous and hottest component of the system, but it is also the less massive one. This is a clear indication that the primary has a larger helium content, thus that it is more evolved, than the secondary.

\subsection{Evolutionary Status}

Our photometric and spectroscopic solution shows that the system has a mass-ratio very close to unity. Twin binaries are expected to be common among high-mass systems with short orbital period (Krumholz \& Thompson 2007). This results from the fact that mass transfer takes place very early in their life. In such a case, the mass transfer starts with a fast Case A, occurring on the thermal timescale of the components, until the system reaches a mass-ratio close to one. At that point, it switches to a slow Case A, which develops itself on the nuclear timescale (Kippenhahn \& Weigert 1967). Given the relative timescales involved, it is more probable that we are observing 

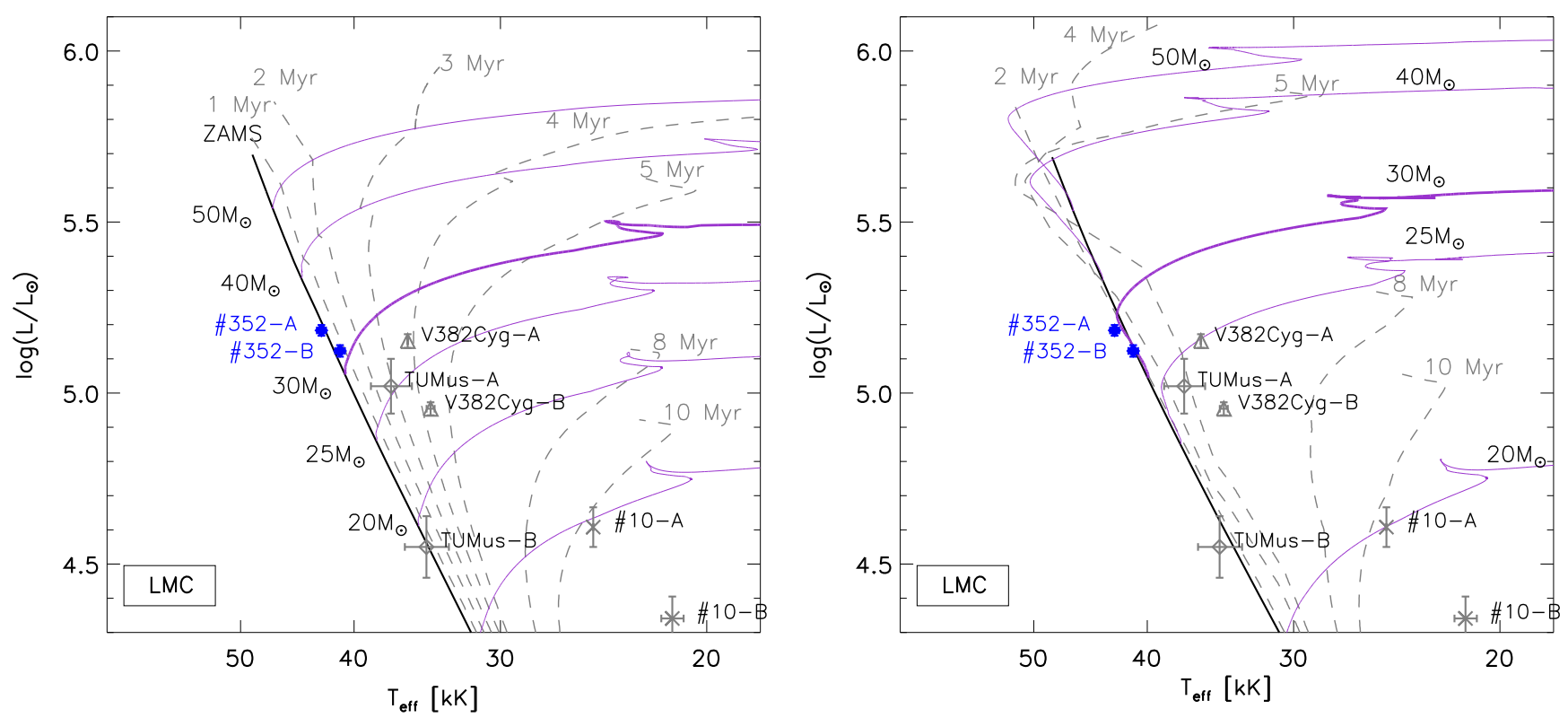

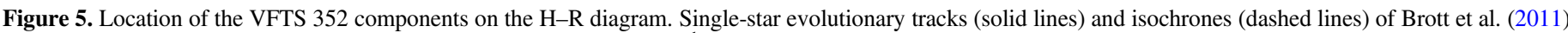

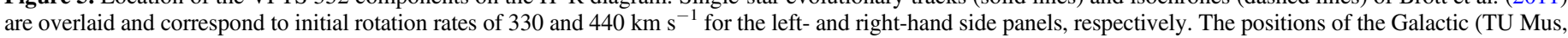
V382 Cyg) and SMC (OGLE SMC-SC10 108086; \#10) contact binaries are indicated for comparison.

VFTS 352 during its slow Case A phase, as already suggesed in Section 3.4.

There are currently no models for massive stars reliably predicting the physical properties of the stars during the overcontact phase. Even though the models by de Mink et al. (2007) computed with the TWIN version of the Eggleton code (Eggleton 2006) allow for the formation of (shallow) contact, they do not include a realistic treatment of heat transfer during the contact phase. For low mass contact binaries this appears to be a crucial ingredient (Yakut \& Eggleton 2005). At today's day, our results can thus not be directly confronted with theoretical binary computations. In an attempt to identify physical processes that are specific to the present configuration, we instead looked for deviations between the properties of VFTS 352 and the predictions of single stellar models.

To obtain a precise interpolation of the single star evolutionary tracks of Brott et al. (2011), we use the Bayesian comparison tool BONNSAI $^{16}$ (Schneider et al. 2014b). We adopt a Salpeter initial mass function and a flat rotational distribution as priors and we provide the temperatures, masses, luminosities and rotation rates that we measured. As can be seen in Figure 5 (lefthand panel), no model can selfconsistently reproduce all the observables at once: the stars appear too hot for their dynamical masses.

Comparing the Bonn's evolutionary tracks with binary results listed in the review of Torres et al. (2010), Schneider et al. (2014b) also found that systems with effective temperatures higher than $25,000 \mathrm{~K}$ tend to be hotter than expected for their dynamical mass, although the difference was of the order of $1000 \mathrm{~K}$ only. While this could indicate a systematic discrepancy between observed and modeled effective temperatures, Schneider et al. (2014b) also suggested that the difference could be due to calibration issues.

In particular, we reviewed the only four O-type binaries listed in Torres et al. (2010) and found that, in the original

\footnotetext{
16 The BONNSAI web-service is available at www.astro.uni-bonn.de/stars/ bonnsai
}

analysis of these systems, the authors used the primary temperature fixed from its spectral type. While this is a logical approach if the distance is a free parameter, it is susceptible to significant bias if a problem occurs in the adopted calibrations. Furthermore, the error will propagate to the secondary star properties as well. Indeed, the calibrations adopted in the original analyses were outdated, e.g., Lamers (1981), Popper (1980), Conti (1973a, 1973b), etc. They carry a systematic shift to higher temperatures $(\sim 1000 \mathrm{~K})$ compared to more recent models which take into account non-LTE, wind, and lineblanketing effects, e.g., Martins et al. (2005). For example, the V3903 Sgr system was analyzed by Vaz et al. (1997). They used Conti (1973a) and Lamers (1981) to fixed the primary effective temperature $T_{1}=38,000 \mathrm{~K}$, for an O7V spectral type. However, an O7V has 36,872 K in Martins et al. (2005), therefore, $\sim 1100 \mathrm{~K}$ lower.

According to this discussion, one would need to re-analyze all hot systems listed in Torres et al. (2010) using modern stateof-the-art calibration and sophisticated models. While this would be an important work, it lays far beyond the present paper. We however emphasis that, in the VFTS 352 analysis, we do not use a fixed primary temperature but adjusted both $T_{1}$ and $T_{2}$, see Section 3.2. As a consequence, our analysis does not suffer from the same drawback as the historical analyses quoted by Torres et al. (2010).

Interestingly, we find that rapidly rotating single star models can reproduce the masses, luminosities and temperatures of the VFTS 352 components. A good match is found adopting initial rotation rates of $450_{-10}^{+70} \mathrm{~km} \mathrm{~s}^{-1}$ (Figure 5, right hand panel). Such high rotation rates are of course ruled out by the widths of the spectral lines in the VLT/FLAMES data and would further not be expected given tidal locking. However, the good agreement between these extreme rotating models and the observed stellar parameters may be interpreted as evidence that the VFTS 352 components have experienced more mixing than comparable single stars, possibly as a result of their binary nature. 


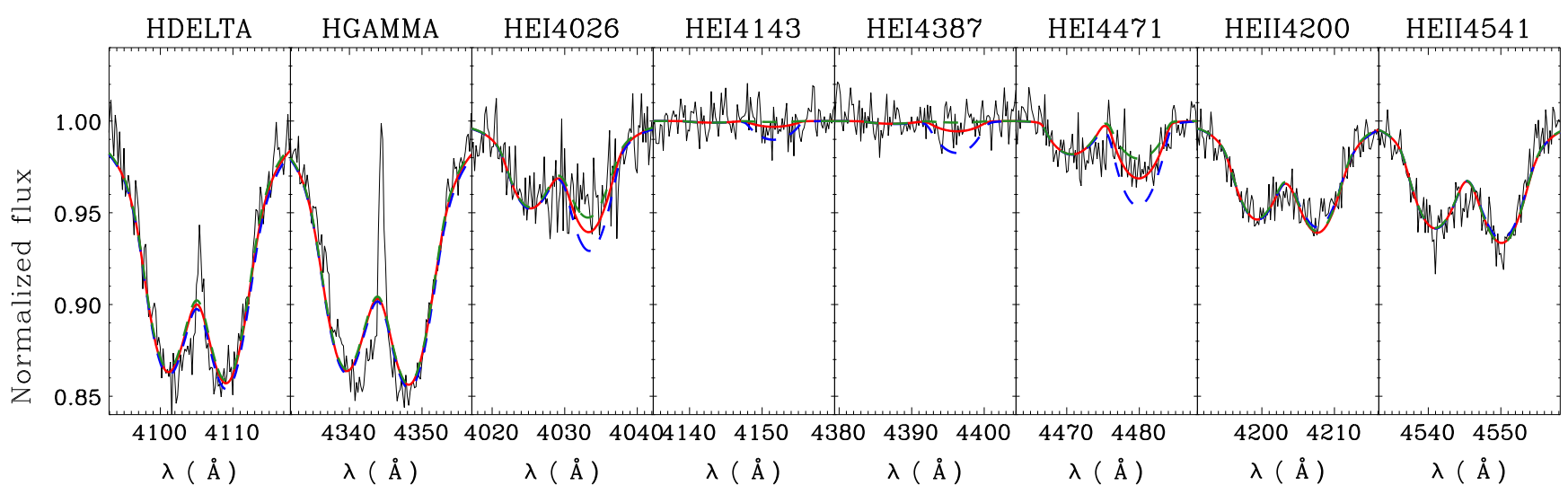

Figure 6. Close up on $\mathrm{H}$ I, He I and He in line profiles in the spectrum of VFTS $352(\phi=0.139)$ compared to FASTwind models computed with the best-fit parameters of Table 1 (plain green line) and with a $\pm 2700 \mathrm{~K}$ difference in the primary effective temperature $T_{1}$ (blue and red dashed lines). While the green curve seems to fit the HeI $\lambda \lambda 4026$ better than the red curve, this helium line is a trace element in such a hot star and strongly impacted by the Struve-Sahade effect as discussed in Section 3.3.

\subsection{Signature of Enhanced Mixing?}

As mentioned in the previous section, the best fitting single star models are those that experienced enhanced internal mixing. The high temperatures and compactness of VFTS 352 can indeed be explained if extra helium produced in the center of the star is mixed throughout the envelope resulting in quasi homogeneous stars (Maeder 1987; Yoon \& Langer 2005). For the single star models of Brott et al. (2011) that we used above, enhanced mixing is a consequence of rotationally induced instabilities. While the single star models are not appropriate for direct comparison, the good match between these fast rotating models and the physical properties of the VFTS 352 components may be a signature that the stars have experienced additional mixing compared to similar single star with a $330 \mathrm{~km} \mathrm{~s}^{-1}$ rotation rate, even if the nature of the additional mixing processes in VFTS 352 may differ from those implemented in single star models.

Stars in close binary systems are generally thought to experience enhanced mixing in comparison with a single stellar models with the same birth rotation rate (e.g., de Mink et al. 2009; Song et al. 2013). For example, (1) tidal locking of the rotation period of the outer stellar layers with the orbital period creates internal shear. The shear can persist as the star evolves and its internal structure slowly changes. As the tides force the stars to stay in synchronous, (2) angular momentum from the orbit is converted into the spin angular momentum of the stars. For single stellar models higher angular momentum implies more internal mixing. If this holds true for binary systems, it would imply enhanced mixing. Finally, (3) the deformation of stars that are very close to filling their roche lobe may also play a role. In single stars the deviation of spherical symmetry is the seed for the Von Zeipel effect and the Eddington-Sweet circulations. For close binary stars the symmetry around the rotation axis is also broken, adding further possibilities for internal processes to enhance mixing.

It is unclear how these processes (and possible additional processes not listerd here) operate and possibly interplay with each other. Attempts to account for (some of) this additional physics were made by de Mink et al. (2009) and later independently by Song et al. (2013). Both groups indeed predict that stars in compact binaries experience more mixing than single stars with identical birth rotation rate. This is consistent with our finding that the best matching single star models have higher initial rotation rates than expected for VFTS 352.

The models by de Mink et al. (2009) further predict enhancements of the surface with $\mathrm{CNO}$ burning products. The rapidly rotating single star models that reproduce our observations imply a surface helium mass fraction of $39 \%$ and $32 \%$ for the primary and secondary components. These indirectly inferred abundances are consistent with the detection limits obtained from our spectra.

\subsection{Alternative Scenarios Without Enhanced Mixing}

An alternative explanation for the high temperatures and increased helium surface abundance that does not invoke enhanced mixing involves stripping of a star from its enveloppe. This exposes the deeper layers which show signatures of the nuclear burning in the center of the convective core. In absence of extra mixing one needs to strip the star(s) down to the layers that were once part of the convective core. The removal of a star's outer layers can be achieved either through stellar winds or by Roche lobe overflow and subsequent mass transfer. Here we briefly discuss these scenarios.

While stellar winds can play a major role in the advanced evolutionary stages of massive stars, we expect them to have had a negligible effect for the relatively unevolved components of VFTS 352. There are no significant wind spectral signatures in the spectrum of VFTS 352 (Walborn et al. 2014). The reduced metallicity of the LMC also implies that the role of stellar winds in this early stage is neglible. We thus conclude that stripping by stellar winds alone is unable to remove a substantial part of the envelope.

The second scenario concerns Roche lobe overflow by the initially most massive star and (partial) accretion by the companion. Allthough some mass loss due to Roche lobe overflow seems likely, it is hard-within our current understanding of binary interaction-to reconcile the VFTS 352 observables using mass transfer alone (i.e., without invoking extra mixing). To explain the surface helium enrichment of the primary star-39\% by mass-in absence of extra mixing, the star needs to shed about half of its total mass. This implies an extreme initial mass ratio $M_{2} / M_{1} \lesssim 0.5$, with the exact value depending on how much the mass gainer accreted. Mass transfer in systems with such extreme mass ratios is expected to 
be highly non-conservative due to the thermal expansion of the mass gainer (Pols 1994; Wellstein et al. 2001; de Mink et al. 2007). Also the current short orbital period of VFTS 352 may be seen as an indication of a prior non-conservative evolution that has resulted in a shrinkage of an originally wider orbit.

Yet, this picture is very hard to reconcile with the inferred helium surface enrichment of the secondary-32\% by mass fraction-which suggests that the secondary has accreted at least some of the primary enriched material. A simple back-of the enveloppe calculation indeed shows that, assuming a primary enriched material with a 39\% He-mass fraction at most, the secondary star should have accreted at least $6 M_{\odot}$ in order to increase the He-mass fraction of its enveloppe from $26 \%$ to $32 \%$. This contradicts the non-conservative nature of the scenario implied by the very low initial mass-ratio and current short orbital period. Reversing the role of the primary and secondary in this scenario does not help, hence mass transfer alone is unable to provide a satisfactory explanation either.

In view of the current state of the models, it seems that neither stellar winds nor Roche lobe overflow, acting alone, are sufficient mechanisms to provide a simple and natural explanation for the inferred properties of VFTS 352. We thus suggest enhanced internal mixing, either as the sole culprit or in combination with the processes above, as the most probable explanation for their current properties.

\subsection{Age Constraints}

If the VFTS 352 components are evolving quasi-homogeneously under the combined effects of rotation and tidally induced enhanced mixing, the position of the stars in the HRD would then remain close to the zero-age main sequence for the first few Myr of their life (Brott et al. 2011). Given our constraints on the temperature and luminosities, we obtain age estimates of $3.4 \pm 0.2$ and $2.4 \pm 0.2 \mathrm{Myr}$ for the primary and the secondary. Both the higher helium mass fraction of the primary and the different age estimates provide indications of a previous mass-transfer event.

The age that we infer opens up to the possibility that the system was not born at its current location but may have migrated from one of the young 30 Doradus clusters after receiving an impulse through dynamical interaction (e.g., Fujii \& Portegies Zwart 2011). The systemic radial velocity, $\gamma=262.8 \pm 1.2 \mathrm{~km} \mathrm{~s}^{-1}$, is in overall agreement with that of the 30 Doradus region and does not indicate that the system is a runaway star along the line of sight. We inspected the OGLE data and, while the dispersion of the measurements is large, a significant proper motion of the order of $-0.8 \pm 0.3$ milliarcsec $\mathrm{yr}^{-1}$ may be present along the declination axis. While error bars are large, such a proper motion allows for the possibility that the system has been ejected from the NGC 2070 association or from R136, the very massive cluster at its core. An ongoing Hubble Space Telescope proper motion program (GO 13359, PI: Lennon) will soon be able to confirm the tentative proper-motion detected in the OGLE data.

\subsection{Final Fate}

Two possible outcomes of the overcontact configuration of VFTS 352 can be envisioned, depending on whether the stars will evolve redward or blueward in the HRD. In the first scenario, the build-up of an internal chemical gradient will reduce the efficiency of the rotationally and (possibly) tidally induced mixing over time. Stars will then switch to a more standard evolution scenario in which secular expansion will push the VFTS 352 components into deeper contact and the system will eventually merge.

While the merging process itself is poorly understood, it may produce an intermediate luminosity transient (Soker \& Tylenda 2006), such as the one associated with the merger of the lower-mass system V1309 Sco (Tylenda et al. 2011). The likely outcome of the merging process is a rapidly rotating and possibly magnetic single star (Ferrario et al. 2009; Langer 2012; de Mink et al. 2013, 2014). According to the models by Yoon et al. (2006) and Brott et al. (2011), such a fast rotating massive star may itself experience significant mixing induced by rotation. A similar system at low metallicity might even retain enough angular momentum to fulfill the requirements of the collapsar scenario (Woosley 1993) for the progenitors of long GRBs as suggested by Yoon \& Langer (2005) and Woosley \& Heger (2006).

In the second scenario, the internal mixing is such that the stars would pursue (quasi) chemically homogeneous evolution. They would become hotter and more luminous, but would remain compact. The system may thus avoid the coalescence phase described above and the stars would retain their tidally locked rapid rotation rate throughout their main sequence lifetime (de Mink et al. 2009). Once most of the stellar hydrogen will have been burned, the VFTS 352 components are then expected to enter a Wolf-Rayet phase and to develop strong stellar winds.

Tides can in principle counteract the spin down induced by mass-loss and preserve the rapid rotation. This however depends on uncertain aspects such as how much the orbit widens during this phase and how efficient the tides are. If the components in VFTS 352 can retain their high spins until the end of their lives they would be interesting candidates as progenitors of long GRBs, despite the relatively highmetallicity environment provided by the LMC.

Regardless of the evolution of the stellar spin, a scenario in which VFTS 352 avoids coalescence implies two massive stars whose cores are enlarged as a result of mixing. In such a configuration, the most likely final outcome of is the formation of two stellar mass black holes. This makes VFTS 352 also interesting as a potential progenitor of binary black hole merger and gravitational wave source.

This work is based on data obtained at the European Southern Observatory under program IDs. 182.D-0222, 090.D-0323, and 092.D-0136. The authors are grateful to the referee for comments that help improving the manuscript, to N.R. Walborn for fruitful discussions and to J. Pritchard and the ESO staff for their support during the observing campaigns. L.A.A. acknowledges support from the Fundação de Amparo à Pesquisa do Estado de São Paulo_FAPESP (2013/18245-0 and 2012/ 09716-6); S.dM., a Marie Sklodwska-Curie Reintegration Fellowship (H2020-MSCA-IF-2014, project ID 661502) awarded by the European Commission; J.M.A., from the Spanish Government through grant AYA2013-40611-P; R.H.B., from FONDECYT No 1140076. This work has been partly supported by the Polish National Science Centre grant No. DEC-2011/03/B/ST9/02573. 


\section{REFERENCES}

Almeida, L. A., Jablonski, F., Tello, J., \& Rodrigues, C. V. 2012, MNRAS, 423, 478

Antokhina, E. A., Srinivasa Rao, M., \& Parthasarathy, M. 2011, NewA, 16,177

Bagnuolo, W. G., Jr., Gies, D. R., Riddle, R., \& Penny, L. R. 1999, ApJ, 527, 353

Brott, I., de Mink, S. E., Cantiello, M., et al. 2011, A\&A, 530, A115

Charbonneau, P. 1995, ApJS, 101, 309

Claret, A., Díaz-Cordovés, J., \& Gimenez, A. 1995, A\&AS, 114, 247

Conti, P. S. 1973a, ApJ, 179, 181

Conti, P. S. 1973b, ApJ, 179, 161

Crowther, P. A., Schnurr, O., Hirschi, R., et al. 2010, MNRAS, 408, 731

de Mink, S. E., Cantiello, M., Langer, N., et al. 2009, A\&A, 497, 243

de Mink, S. E., Langer, N., Izzard, R. G., Sana, H., \& de Koter, A. 2013, ApJ, 764, 166

de Mink, S. E., Pols, O. R., \& Hilditch, R. W. 2007, A\&A, 467, 1181

de Mink, S. E., Sana, H., Langer, N., Izzard, R. G., \& Schneider, F. R. N. 2014, ApJ, 782, 7

Değirmenci, Ö. L., Sezer, C., Demircan, O., et al. 1999, A\&AS, 134, 327

Díaz-Cordovés, J., Claret, A., \& Gimenez, A. 1995, A\&AS, 110, 329

Eggleton, P. (ed.) 2006, in Evolutionary Processes in Binary and Multiple Stars (Cambridge: Cambridge Univ. Press)

Eggleton, P. P. 1983, ApJ, 268, 368

Evans, C. J., Taylor, W. D., Hénault-Brunet, V., et al. 2011, A\&A, 530, A108

Ferrario, L., Pringle, J. E., Tout, C. A., \& Wickramasinghe, D. T. 2009, MNRAS, 400, L71

Fujii, M. S., \& Portegies Zwart, S. 2011, Sci, 334, 1380

Gilks, W. R., Richardson, S., \& Spiegelhalter, D. J. E. 1996, Markov Chain Monte Carlo in Practice (London: Chapman and Hall)

Hilditch, R. W., Howarth, I. D., \& Harries, T. J. 2005, MNRAS, 357, 304

Kippenhahn, R., \& Weigert, A. 1967, ZAp, 65, 251

Krumholz, M. R., \& Thompson, T. A. 2007, ApJ, 661, 1034

Kudritzki, R.-P., \& Puls, J. 2000, ARA\&A, 38, 613

Lamers, H. J. G. L. M. 1981, ApJ, 245, 593

Langer, N. 2012, ARA\&A, 50, 107

Leung, K.-C., \& Schneider, D. P. 1978, ApJ, 222, 924

Linder, N., Rauw, G., Sana, H., de Becker, M., Gosset, E., et al. 2007, A\&A, 474, 193
Lorenzo, J., Negueruela, I., Baker, A. K. F. V., et al. 2014, A\&A, 572, AA110

Maeder, A. 1987, A\&A, 178, 159

Maíz Apellániz, J., Evans, C. J., Barbá, R. H., et al. 2014, A\&A, 564, AA63

Martins, F., Schaerer, D., \& Hillier, D. J. 2005, A\&A, 436, 1049

Mason, B. D., Hartkopf, W. I., Gies, D. R., Henry, T. J., \& Helsel, J. W. 2009, AJ, 137, 3358

Palate, M., \& Rauw, G. 2012, A\&A, 537, AA119

Penny, L. R., Ouzts, C., \& Gies, D. R. 2008, ApJ, 681, 554

Podsiadlowski, P., Joss, P. C., \& Hsu, J. J. L. 1992, ApJ, 391, 246

Popper, D. M. 1978, ApJL, 220, L11

Popper, D. M. 1980, ARA\&A, 18, 115

Pols, O. R. 1994, A\&A, 290, 119

Puls, J., Urbaneja, M. A., Venero, R., et al. 2005, A\&A, 435, 669

Sana, H., de Koter, A., de Mink, S. E., et al. 2013, A\&A, 550, A107

Sana, H., de Mink, S. E., de Koter, A., et al. 2012, Sci, 337, 444

Sana, H., Le Bouquin, J.-B., Lacour, S., et al. 2014, ApJS, 215, 15

Schneider, F. R. N., Izzard, R. G., de Mink, S. E., et al. 2014a, ApJ, 780,117

Schneider, F. R. N., Langer, N., de Koter, A., et al. 2014b, A\&A, 570, A66

Soker, N., \& Tylenda, R. 2006, MNRAS, 373, 733

Song, H. F., Maeder, A., Meynet, G., et al. 2013, A\&A, 556, A100

Sota, A., Maíz Apellániz, J., Morrell, N. I., et al. 2014, ApJS, 211, 10

Torres, G., Andersen, J., \& Giménez, A. 2010, A\&AR, 18, 67

Tylenda, R., Hajduk, M., Kamiński, T., et al. 2011, A\&A, 528, A114

Udalski, A., Szymanski, M. K., Soszynski, I., \& Poleski, R. 2008, AcA, 58,69

Vaz, L. P. R., Cunha, N. C. S., Vieira, E. F., \& Myrrha, M. L. M. 1997, A\&A, 327, 1094

Vink, J. S., de Koter, A., \& Lamers, H. J. G. L. M. 2001, A\&A, 369, 574

Walborn, N. R., Sana, H., Simón-Díaz, S., et al. 2014, A\&A, 564, A40

Wellstein, S., Langer, N., \& Braun, H. 2001, A\&A, 369, 939

Wilson, R. E. 1979, ApJ, 234, 1054

Wilson, R. E. 2001, IBVS, 5076, 1

Wilson, R. E., \& Devinney, E. J. 1971, ApJ, 166, 605

Woosley, S. E. 1993, ApJ, 405, 273

Woosley, S. E., \& Heger, A. 2006, ApJ, 637, 914

Yakut, K., \& Eggleton, P. P. 2005, ApJ, 629, 1055

Yoon, S.-C., \& Langer, N. 2005, A\&A, 443, 643

Yoon, S.-C., Langer, N., \& Norman, C. 2006, A\&A, 460, 199 